\title{
INHALTS-ANZEIGE.
}

\section{ERSTER BAND.}

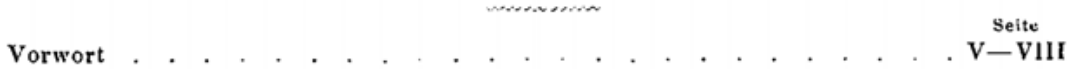

Erstes Kapitel.

Reise nach Konstantinopel.

Pesth und Ofen - Fahrt auf der Donau - - von Neusatz bis Orsova - Orsova, Melıadia - Mchadia - Abreise von Orsova - Fahrt auf der Donau bis Giurgevo - von Giurgevo nach Galatz - Abreise von Galatz - Sulina - Fahrt auf dem schwarzen Meere - der Bospurus bis Konstantinopel . . . . . $1-16$

Zweites Kapitel.

\section{Aufenthalt in Konstantinopel.}

Konstantinopel, Râmis Bey -- der Ranaçân - Türkische Mahlzeit - Kriegrsschule, Schewket Pascha - Atmeidân n. s. w. - Türkisches Haus, Sclaven - der Sultan, Balykly -- Kaserne, Kara Gös - Leanderthurm, Kadhikjoe - Jenikjoe, Therapia, Bujukdere - die siissen Wasser, das neue Mnseum - das neue Serai, Besuch bei Schewket Pascha. . . . . . . . . . . . . . . . . . 17-33

Drittes Kapitel.

Reise von Konstantinopel bis Damascus.

Abreise von Konstantinopel, dic Dardancllen, Tenedos -- Lesbos, Smyrna - Chios, Samos, Ikaria - Kos, Rhodus - Rhodus, Hedenborg, Cypern - Jeirút -Abreise, der Libanon - Uebcrnachtung in dem neuen Chân - der Libanon, dic Beqâa - Beduinen - Ritt über den Antilibanon nach Hâme - Anblick von Damascus . . . . . . . . . . . . . . . . . . 34-53

Viertes Kapitel.

Damascus.

Damascus, Häuser - Leben der Muhammedaner - Hoffmann, Leben der Muhammedaner - Graf Guyon - Baron Splenyi, Feizy Bey (Kollmann) - Abreise des Hadsch - Ibrahim Pascha - Landpartie, Dschobar - Diner bei Meliemed Pascha - jüdische Verlobung - Militär-Lazareth - neıe Wohnung - Temperatur, Eis - der Bárada, Gärten - Reise nach Malûla - Berse, Máraba, Sidaaya Dawâni, Dreschwagen, 'Ain Tîne - Malûla - Pistacien, Menîn - Damascus, die Drusen - Ibrahim Pascha - Kampf mit den Drusen - Muhammed Dâwud Abbas Scheref - Diner bei einem Drusen - Drusen - türkisches Bad - die Dscherde - Fahne Muhammed's - Abendgeselischaft bei Mr. Wood - Comte d'Escayrac - Witterung - Elias, Paulus - - Eintheilung der Stadt, Verwaltung Bevölkerung - Umaijaden-Moschee - Muhammedaner - Characterzüge der Araber - Christen - Nestorianer, Kopten - Jakobiten, Leichenbegänguiss Armenische Cbristen - Orthodoxe Griechen - Melchiten - Messe - Epiphanias - Taufe - Verlobung - Hochzeit - Begräbniss - Gebräuche der Melchiten - andere unirte Christen - Syrianer - Maroniten - Weihnachtsfest der 
Maroniten - orientalische Christen - Römische Katholiken, Franciscaner Capuciner - Jesuiten - Lazaristen - evangelische Missionare - evangelische Christen, Juden - jüdische Hochzeit - Drusen - Trachten - Tracbten der Männer - Trachten der Frauen - Schönheitsmittel - Spiele - Kinderspiele Spiele der Erwachsenen - Schachspiel - Damenspiel, Kartenspiele - Mánqale Musik - Qara Gos - Raqi - andere Getränke - Brod, Brodbacken - Speisen - Sitten und Gebrăuche der Araber, der Orientalen überhaupt . . . 54-174

Fünftes Kapitel.

Reise von Damascus nach Jerusalem.

Abreise von Damascus - Dêreje, Steuern - Dschedêde, Kátana - Raschâya, die Familie Schehâb - Rachâya, Weiterreise - Hasbâya, Dr. Hanna - Emir As'ad, Weiterreise - Bint el Dschebêl - Safed - Mágdala, Tiberias - Der Tiberias." sce, der Berg der Seligkeiten, Lubi - Kana, Nazareth - Berg Tabor, Ebenc Esdrêlom , Dschenîn - Nablûs - Jerusalem . . . . . . . . . . 175-196

\section{Sechstes Kapitel.}

\section{Jerusalem und Umgegend.}

Jerusalem, Lage der Stadt, Omar-Moschee - Beschreibung der Stadt - Grabeskirche - das griechische Kloster - Grabeskapelle - Häuser - Umgebung der Stadt - der Oelberg - Teiche, Begräbnissplätze - Witterung - Charfreitag, Bekanatschaften - Prctestanten, Missiozare - Katholiken - Kreuzeskloster, griechische Christen - Armenier - Bibliothek des armenischen Patriarchats Jakobiten - Nestorianer, Abyssinier - Kopten - Juden, der grosse Sabbath Chasidim, Karäer - Bevölkerung des Paschaliks - Ausflug nach Nablûs Sebaste (Samarîa) - Nablûs, Pesachfest der Samaritaner - Rückkehr, Pilgerzug nach dem Jordan - 'Ain es Sultân (Quelle des Elîsa) - Lager an der Quelle, Hitze, Aufbruch am Morgen - Jericho, Baden im Jordan, das todte Meer - das griechische Kloster Mar Saba - Jerusalem, Fusswaschung, Charfreitag - das heilige Feuer - (Mizpa) Neby Samuêl - Jahresfeier des Diakonissen-Hauses Spaziergang nach Bethlehem - Bethlehem - Ausflug nach Jaffa - Lud (Lydda), Ramle - Jaffa - deutsche Ansiedler - Jaffa, Rückkehr nach Jerusalem Abstecher nach Hebron - Hebron - Abraham's Eiche, Rückkehr . . . 197-259

Siebentes Kapitel.

\section{Reise nach Nablûs und Aufenthalt daselbst.}

Abreise nach Nablûs - von Jerusalem bis Bîre - von Bîre bis Nablùs, Bevölkerung von Nablûs - Industrie, Verwaltung von Nablûs - Brief beförderung - Häuser von Nablûs - Klima von Nabluss, die Samaritaner - Geschichte der Samaritaner - Correspondenz mit ihnen - rothe Turbane - Haartracht der Priester, weibliche Tracht - Beschäftigungen - Gebräuche, Geburt, Beschneidung, Entwöhnung, Verheirathung - Bigamie, Leviratsehe - Ehescheidung, Begräbniss Glaube, kein Bilderdienst, geheimnissvolles Gemach - Engel und Teufel, Messias - jüngstes Gericht, Paradies, Hölle - die fünf Bücher Mosis - heilige Stellen auf dem Garizim - Pesachfest - Fest der ungesäuerten Brode - andere Feste - Versammlungstage - Priester . . . . . . . . . . . . 260-292

Achtes Kapitel.

\section{Reise von Nablûs nach Damascus.}

Abreise von Nabluts, Baka - Karmelgebirge, Kloster auf dem Karmelgebirge Acca - geographische Notizen, vou Acca nach Sûr (Tyrus) - Hiram's Grab --. Sûr (Tyrus) -- Weg nach Saida (Sidon) - Neby Jûues, Beirút, Kameele, Mehemed 'Aly - Reise nach Damascus - Bekfâya, Salıle - Sebdâny, Brodbacken, Sûq Bárada - Damascus . . . . . . . . . . . . . . . . . 293-306

\section{Neuntes Kapitel.}

Reise von Damascus auf den Libanon und nach Beirút.

Chelbûn - Weiterreise, Bludân - Birdân oder Bridàn, die Ruinen von Baalbek die Stadt Baalbek - Abreise von Baalbek, Deir el ahmar - die Cedern des 
Libanon - das Kloster Qes'háya - Ehden - Karmeliter-Kloster, Chasrûn, Dimân - der maronitische Patriarch — die Maroniten - das Kloster Qanobîn -Tarábolus (Tripolis) - Batrûn - Dschebêl (Byblus) - Ghasîr, Klöster - Ausflug nach dem Libanon -- katholisch-armenischer Katholikos - Mar Schalita, Der Scherfe - zweiter Ausflug in den Libanon - Dêr Meifûq - Tadmor - Aqûra Jenuch - Mneitre - allgemeine Bemerkungen - Trachten, Beirút . . 307-343

\section{Zehntes Kapitel.}

Reise nach Cilicien und Cypern.

Larnaka, Ladakîa - Iskenderûn - Mersîn, Wagen von Büffeln gezogen - Tarsus Chân in Tarsus - Ueberreste in Tarsus - Mr. Clapperton - Casino, Abreise nach Adana - Adana, armenische Protestanten - Rïckkehr nach Tarsus und Mersîn - Soli (Pompejopolis) - Abreise nach Cypern - Larnaka - erste Tour auf der Insel - Mat̂sa (Famagusta) - St. Sergis (Sergius) - Schlangen, Sálamîn - zweite Tour auf der Insel - Nikósia (Levkósia) - Dikomo, belli paêsi Tzerinia - Lampt̂sa - Rückkehr nach Larnaka - Rückkehr nach Beirût 344-374

Ueber die Drusen (Anm. 30 zu S. 148) . . . . . . . . . . . . . 375-408

\section{ZWEITER BAND.}

\section{Erstes Kapitel. \\ Reise von Beirút bis Háleb,}

Abreise, Batrûn, Tarábolus - Hafen von Tarábolus, Ladakîa, Iskenderûn Bailân - Armenier, Römerstrasse, Sôúk Sù - T'urkmanen, 'Ain beidhâ - Turmauîn, Ruinen, Háleb - Handel, Häuser - Fanatismus der Moslemen - statistische Angaben, Christen - alte Synagoge, armenische Kirche - armcnische Taufe, Antiken, Codices - katholische Klöster, Auszug von Basch bosúk's . . . 1-13

\section{Zweites Kapitel.}

Reise von Háleb bis Süérek.

Achterîn (Tachterîu), Sambûr - Nisib, Sari kodsch - Biredschik - Abreise von Biredschik, Merbi - das Gebirge el Malakije (el Malatije), Basalt — Hawank (Hawak) - Treulosigkeit der kurdischen Begleiter - Qara Tschurûn, Weiterritt - Süwérek (Süérek) . . . . . . . . . . . . . . . . . . . 14-25

\section{Drittes Kapitel.}

Reise von Süérek bis Máredîn.

Qara Baghdsche - Qáradscha Tâgh, Diarbékir - Weiterritt - Cháneke bis Máredîn - Máredin - Aufstand, Beduinen - Beduinenstämme . . . . . . 26-37

\section{Viertes Kapitel.}

\section{Reise von Máredîn bis Môsul.}

'Amûda, Dâra, Hamdûni - Kurden, Weiterreise - Nisibîn - Tell Dschihân, Weiterreise - Scheich 'Aly - Kodschar, Kurden, Dschesîre - D schesîre -. Dschebel Dschûdi, Dakejan ('Takejan) - Sacho - Tarkeschan, Tülüb - Amêdi (Amêri), Chàttără - Tell Esköf, Ankunft in Môsul . . . . . . . . . . . . 38-51

\section{Fünftes Kapitel.}

Reise von Môsul bis Bagdâd.

Anfertigung des Kelek (Bootes) - Abfahrt, Neby Junos, Ausgrabungen - Weiterfahrt, Beduinen - Dschebel Makchûl, Beduinen - Dschebel Hemrîn, Tekrît - 
Imâm Tôr, Eski Bagdâd - Ziehbrunnen, Imâm es Sâmera (Sermenra) u. s. w. Weiterfahrt bis Bagdâd - Bagdâd, Mr. Brühl, Col. Rawlinson - Abreise von Bagdâd . . . . . . . . . . . . . . . . . . . . . . . . . . . $52-66$

Sechstes Kapitel.

\section{Reise von Bagdâd bis Sûq esch Schiuch.}

Chân Iskenderije, Weiterritt bis in die Gegend von Hille (Babylon) - Hille (Babylon) - Thurm von Babel - Fahrt auf dem Euphrat nach Diwanije - Diwanije Abfahrt von Diwaníje - Fahrt bis Samawât - Samawât - Weiterfahrt, strohhütten - Fahrt nach Sûq esch Schiuch - Ankunft in Sûq esch Schiuch . . 67-82

Siebentes Kapitel.

Aufenthalt in sûq esch Schiuch.

St̂q esch Schiuch - der Chân - Scheich Jahja - Witterung - Klima - Tracht Besuch bei Mansur, dem Scheich der Montefik - Besuch bei dem Priester Jahja, die Mandäer - Namen der Mandäer ... Legenden der Mandïer - Priester der Mandïer - Tempel, Taufe - Verlobung, Trauung - Leichenbegängniss Trachten - Speisen u. s. w. - Teufelbeschwörung, Execution - meine Lebensweise in Sûq esch Schiuch, Brombeeren - Kinder-Procession, Geflügel - Fische, Ungeziefer - Getraide - Töpfer, Ziegelofen, Erdsalz - verschiedeue Ansichten der Muhammedaner - verschiedene Secten, allerhand Mittel - Besuch von Mr. Taylor - Witterung - Bedrückung, Abreise . . . . . . . . . . 83-137

Achtes Kapitel.

Rückreise von Sûq esch Schiuch nach Bagdâd.

Abfahrt von Sûq esch Schiuch - Diwanije, Vögel - Pflanzen, Weiterfahrt - Hille, Bagdâd - Bagdâd . . . . . . . . . . . . . . . . . . . 138-148

Neuntes Kapitel.

Reise von Bagdâd nach Schirâs.

Ktesiphon, Kût el Ammâra - Esra's Grab, Kût - Basra - Abfahrt von Basra Ankunft in Buschihr - Trachten, Temperatur - Abreise von Buschihr - Ritt bis Achmedije - Barasgûn - von Barasgûn his Dahlekije - Weiterreise von Dahlekije - Konar Tacht - Kômaredsch - Kâserûn - Besuch der Synagoge Unannehmlichkeiten - Kotáli Dochter - Weiterreise nach Kotáli Piri San Kotáli Piri San, Sturz vom Pferde - Dostardschûn, Chûni Senijûn - Schiràs, Erdbeben - Folgen des Erdbebens . . . . . . . . . . . . . 149-172

Zehntes Kapitel.

Aufenthalt in Schirâs.

Schirâs - Dr. Fagergrün, Mirza 'Aly - Juden - Gräber von Hâfis und Sâ 'di Rosen - Rosenwasser, Geschenke von dem Prinzen - Chôdscha Petros, ein Parsi - Besuch bei dem Prinzen - der Prinz-Gouverneur -- hoher Beamter langes Frühstück. . . . . . . . . . . . . . . . . . . . . . 173-184

Elftes Kapitel.

Reise über Persepolis und Murghâb nach Jesd.

Abreise von Serqûn nach Persepolis - Persepolis - Nakschi Redscheb, Nackschi Rustem - Nakschi Rustem - Abreise, Siwend, Kawâmabad - Mâderi Suleimân, (Grab des Cyrus) - Ruinen von Murghâb, Châni Kerghân --- Tscheschmêï Guschti, Dschâhi sijâh - Erdi, Dreschwagen, Aberkuh -- Schahre, Schemsabad, Dehschîre 'Alyabad, Chorâscha (Ferâscha) — Tafft, Parsi's, Jesd . . . . . . . 185- 202

Zwölftes Kapitel.

Aufenthalt in Jesd.

Jesd - Parsi's - Bevölkerung - Hindu's - Juden, Muhammedaner - Heirathen auf Zeit, Qatirdschi's -- Diner bei den Parsi's . . . . . . . . . . 203-210 
Dreizehntes Kapitel.

Reise von Jesd nach Ispahân.

Meibûd, Bîdabad, Unterhaltung mit einem Sêid - Bîdabad - Weiterreise, 'Aghda Derwisch, Abreise von 'Aghda - No gumbeh, Laghre - Kûpa - Sekse - Ankunft in Ispahân - Ispahân . . . . . . . . . . . . . . . . . . 211-220

Vierzehntes Kapitel.

Dschulfa und Ispahân.

Dschulfa, Armenier - Bad - gesundes Klima - Fruchtbarkeit - Jahreszeiten Eis, Ispahàn - Bücherhandel -- Betrïgereien - Bibliothck Timur's in Samarcảnd - Minarèi tschambàn - Ritt in die Ungegend von Isp̣ahân . . . 221 - 235

Fünfzehntes Kapitel.

Reise von Ispahân nach Hamadân.

Der Muschtehid -. Weiterreise - Schalisîa oder Kalisîa - Dehâk, Rahmetabad Chombidsch, Kulpagûn (Kulpadgûn) - Chômên, Rabat, -- Chorêmabad, Sèr sachtê - Ser sachtê und Umgegend - Tejnö, Hesâr -- Tûreh, Sengeneh Daulétabad - Mengâvi (Menjủn) - Hamadân - das Grabmal von Esther und Mardochai . . . . . . . . . . . . . . . . 236-251

Sechzehntes Kiapitel.

Reise von Hamadân über Kermanschâh nach Kerind.

Kengawâr - Sahàna -... Bisutûn - Bisutûn - Kermanschâh - Besuch bei dem Prinzeu - T'akki Bostán - Unannehmlichkeit in Kermanschâh - Abreise von Kermanschài - Harûnabad Ankunft in Keriud - Kerind, 'Aly ilảhi's - Obst, Backen von Mazzoth . . . . . . . . . . . . . . 252-264

Siebzehntes Kapitel.

Reise von Kerind bis Bagdâd.

Serpûl -.. Qasri Schirin -- Chánekin, Feth 'Aly Schâh - Anecdoten, Aglıa Atuhamıed Chân -.-- Persische Miinzen, der Quarantaine-Arzt -- die Chaibar -persisclier Wahrsager, Chánekin, Münzen - Weiterreise - Qásrabad (Qisil Robat), Schehraban -- Bakuba . $265-276$

Achtzehntes Kapitel.

Aufenthalt in Bagdâd.

Bagdâd, Aufstand, Mirza - Spaziergang - Lage und Eintheilung ... Bevölkerung -Babi's, Strassen, Häuser - persische Truppen - Pilger, Unsicherheit -- Aufstand der Kurden - Witterung, Process - Verheirathung der Moslemin -. mulainmedanische Hochzeitsfeier - Eberjagden - Col. Rawlinson - Cap. Jones -Mes'ud Béy - Fresuel, Dr. Oppert - Reschid Pascha - Kurden -.. Reschid Pascha, Dr. Duthieul - Mr. Brühl - Solaib-Araber - Kurden, Muhammedaner Gebräuche u. s. w., Aberglaube - Recepte - Haschîsch, Klima --- Spiele - Masse und Gewichte -- Geld - Rückreise nach der Heimath . . . . . . . 277-310

Neunzehntes Kapitel.

Reise von Bagdâd bis Môsul.

Abreise von Bagdâd, Dschesâne, Jenîdsche - Nahrewàn, Jenîdsche - Chormadû, Tâtûk - Kerkúk, chaldäische Christen -- Derwische - Kurden - Altûn Köprü gefangene Kurden - Erbil (Arbêla), Uebergang über den grossen Zàb - der Ghasîr, Dschakülle - Ankunft in Môsul . . . . . . . . . . . . 311-324

\section{Zwanzigstes Kapitel.}

Aufenthalt in Môsul.

Amerikanische Missionare - Bevölkerung - beabsichtigter Aufstand - Jesdenschir Béy - Mr. Rassam - die Jesîdi's - Kujundschúk - Abreise . . . . 325-336 
Einundzwangzigstes Kapitel.

Reise von Môsul bis Orfa.

Abreise - Wüste - Tschillagha, Atschâna - Kemerké, Dûne, Nisibîn - Nisibîn - Dâra - das Kloster Dêr Sa'ferân - Qal'at Marra - Kosar - W'eiterreise - Gewitter - Gebirge - Aufhören des Basalts - Ankunft in Orfa 337--352

Zweiundzwanzigstes Kapitel. Aufenthalt in Orfa und Reise bis Háleb.

Orfa (Edessa) - Reise von Orfa bis Biredschik - Weiterreise von Biredschik nach Háleb - Háleb . . . . . . . . . . . . . . . . . . . . 353-360

Dreiundzwanzigstes Kapitel.

Háleb, und Reise von da über Beirút bis Alexandrien.

Háleb - Missionare - Abreise - Dâna u. s. w. - Antákia (Antiochien) - Iskenderûn, Beirút, Alexandrien . . . . . . . . . . . . . . . . . 361-369

Vierundzwanzigstes Kapitel.

Aufenthalt in Aegypten (Alexandrien und Cairo) und Rückreise nach Triest.

Fahrt nach Cairo - Cairo - Gräber - Die Patriarchen - On (Heliopolis), Obelisk - koptische Kirche - Schubra - Park, Nilinsel - Ritt nach den Pyramiden - die Sphinx - Joseph's Grotte, Moschee in Alt - Cairo - Versteinerter Wald - Rückfahrt nach Alexandrien - Ankunft in Triest . . . . . . 370-387

\section{Anmerkungen zu dem 1. Bande.}

Anmerkung 1-22. - 23. Pilgerreise - 24. das antiochenische Patriarchat 25-29. - 31. das Pferd - der Esel - der Maulesel - das Kameel - das Rindvieh - der Büffel - das Schaf - die Ziege - andere Thiere - über den Gesundheitszustand von Damascus - kurze Uebersicht - das Hospital der aussätzigen Christen - das Hospital der aussätzigen Mubamınedaner - der Aussatz Ursachen - das Militär-Hospital - das Irrenhaus - Schlussbemerkung, die Producte der Türkei verglichen mit denen von Anerica - Anmerkung 32-44. Inschriften . . . . . . . . . . . . . . . . . . . 388-446

Anmerkungen zu dem 2. Bande.

Anmerkung 45. - 46. die Mandäer - Religion der Mandäer - Wohnsitze und Anzahl der Mandäer - Mandäer im Libanon, Ketzer - Eschatologie und Moral der Mandäer - Zeiteintheilung der Mandäer - Feste der Mandäer - das grosse Tauffest - Priestergrade der Mandäer - 47-48. Insecten - 49-50. Pflanzen $51-52$. Inschriften . . . . . . . . . . . . . . . . . . . . . 448-471 